\title{
Endocrine disruptors and female cancer: Informing the patients (Review)
}

\author{
LINO DEL PUP ${ }^{1}$, ALBERTO MANTOVANI ${ }^{2}$, AMALIA LUCE $^{3}$, CARLA CAVALIERE $^{4}$, \\ GAETANO FACCHINI ${ }^{4}$, RAFFAELE DI FRANCIA ${ }^{5}$, \\ MICHELE CARAGLIA $^{3}$ and MASSIMILIANO BERRETTA ${ }^{6}$
}

${ }^{1}$ Division of Gynecological Oncology, CRO Aviano, National Cancer Institute, I-33081 Aviano; ${ }^{2}$ National Institute of Health, I-00161 Rome; ${ }^{3}$ Department of Biochemistry, Biophysics and General Pathology, Second University of Naples, I-80138 Naples; ${ }^{4}$ Division of Medical Oncology, Department of Uro-Gynaecological Oncology; ${ }^{5}$ Hematology-Oncology and Stem Cell Transplantation Unit, Istituto Nazionale Tumori 'Fondazione Giovanni Pascale', IRCCS, I-80131 Naples;

${ }^{6}$ Department of Medical Oncology, CRO Aviano, National Cancer Institute, I-33081 Aviano, Italy

Received February 24, 2015; Accepted March 26, 2015

DOI: $10.3892 / o r .2015 .3997$

\begin{abstract}
Pollutants altering the endocrine system, known as endocrine disruptors (ED), may modify the risk of female cancers. The carcinogenic effect of ED on humans has been confirmed by experimental studies for various substances including pesticides, DDT, dioxins, phthalates, bisphenol A, diethylstilbestrol, as well as heavy metals, but it is difficult to quantify precisely for several reasons hereby reviewed. Carcinogenesis is a complex and multifactorial mechanism that manifests itself over a long period of time, making difficult the detection of the specific contribution of the pollutants, whose absorbed dose is often unknown. The combined effect of various substances leads to complex interactions whose outcome is difficult to predict. These substances may accumulate and carry out their harmful effect on critical periods of life, probably also at doses considered harmless to an adult. ED can also have epigenetic adverse effects on the health of future generations. In conclusion, the carcinogenic effects of endocrine disruptors on female cancer types is plausible although additional studies are needed to clarify their mechanisms and entities. In the last part of the review we suggest ways to reduce ED exposure as it is mandatory to implement necessary
\end{abstract}

Correspondence to: Professor Michele Caraglia, Department of Biochemistry, Biophysics and General Pathology, Second University of Naples, Via L. De Crecchio 7, I-80138 Naples, Italy

E-mail: michele.caraglia@alice.it

Dr Lino Del Pup, Division of Gynecological Oncology, CRO Aviano, National Cancer Institute, Via Gallini 2, PN, I-33081 Aviano, Italy E-mail:1delpup@cro.it

Key words: female cancer, endocrine disruptors, pesticides, DDT, dioxins, phthalates, bisphenol A, diethylstilbestrol, heavy metals, cancer prevention measures to limit exposure, particularly during those periods of life most vulnerable to the impact of oncogenic environmental causes, such as the embryonic period and puberty.

\section{Contents}

1. Endocrine disruptors: Definition by international bodies

2. Endocrine disruptors and carcinogenicity

3. Difficulties involved in studying the carcinogenic effect of endocrine disruptors

4. Models of ED carcinogenesis associated with female tumors

5. Prevention or limitation of ED exposure

6. Considerations for future investigations

7. Conclusion

\section{Endocrine disruptors: Definition by international bodies}

According to the definition of the Endocrine Society, an endocrine disrupting (ED) chemical is an exogenous chemical, or mixture of chemicals, that can interfere with any aspect of hormone action (1). This definition considers the endocrine interference, but does not suggest that all chemicals interfering with any aspect of hormone action have a clinically significant effect. Disease risk depends on the dose, timing of exposure and potency of the chemical. United States Environmental Protection Agency definition is more detailed: 'An ED is an exogenous agent that interferes with the production, release, transport, metabolism, binding, action, or elimination of natural hormones in the body responsible for the maintenance of homeostasis and the regulation of developmental processes' (2).

The World Health Organization (WHO) definition of ED includes the 'consequential' adverse effect: 'An ED is an exogenous substance or mixture that alters function(s) of the endocrine system and consequently causes adverse effects in an intact organism, or its progeny, or (sub)populations' (3). 
Table I. Reasons for the difficulties encountered in studying the ED carcinogenic effect.

$\begin{array}{ll}1 . & \text { ED carcinogenesis is complex, multifactorial and long lasting } \\ 2 . & \text { Lifelong exposure is very difficult to evaluate } \\ 3 . & \text { Extremely low ED doses may interfere, even if they are no longer absorbed, as they are stored in the fat tissue } \\ 4 . & \text { The exposure to a multitude of ED generates many complex and unpredictable interactions } \\ 5 . & \text { The ED can induce carcinogenic effects in vulnerable developmental periods } \\ 6 . & \text { The epigenetic effects of ED can also affect future generations } \\ 7 . & \text { Some individuals may have a particular susceptibility to ED carcinogenesis } \\ 8 . & \text { ED may interfere with environment-genome interactions } \\ 9 . & \text { The competing interests may limit research and public information on ED effects } \\ 10 . & \end{array}$

ED, endocrine disruptors.

Furthermore, the European Union definition of ED states: 'An ED is an exogenous substance that causes adverse health effects in an intact organism, or its progeny, secondary to changes in endocrine function. A potential ED is a substance that possesses properties that may be expected to lead to endocrine disruption in an intact organism'. Therefore, the European definition is close to that of WHO, since it suggests that an ED can induce adverse affects, otherwise a substance should be considered as a potential ED (4).

EDs are a heterogeneous group of chemicals, comprising persistent contaminants [e.g., dioxins, polychlorinated biphenyl (PCB) and brominated flame retardants], pesticides (e.g., triazoles, dicarboximides and triazines), industrial substances (e.g. phthalates and bisphenol A) and natural substances (e.g., phyto-oestrogens). EDs have various effects and mechanisms (e.g., interaction with nuclear receptors and enzyme inhibition). Additionally, susceptibility is modulated by gender and markedly enhanced during prenatal and postnatal development (5).

\section{Endocrine disruptors and carcinogenicity}

Numerous toxicological studies, on laboratory animals and in vitro, as well as human epidemiological trials have been conducted to determine whether environmental ED contribute to increasing the risk of female endocrine-associated cancers, particularly breast and endometrium cancers. It has been suggested that ED hormonal interference partially explains the growing incidence of such types of cancer, which cannot be fully clarified by other known risk factors and mechanisms (6).

As an example, a growing number of well-designed epidemiological and molecular studies have provided substantial evidence that pesticides used in agricultural, commercial, home and garden applications, are associated with excess cancer risk. The increased risk has been observed mainly in people applying the pesticide, although some studies have also identified exposure of bystanders, particularly following exposure during vulnerable lifestages (7).

Individual cancer risk comes from the complex interplay between many factors, including genetics, lifestyle, diet, endogenous hormone status and environmental factors, as well as timing. The perinatal period and the period between age at menarche and first full-term pregnancy may be particularly important for tumor development and latency. Mounting evidence that exposure to even very small amounts of exogenous agents, especially during the crucial stages of human development, is capable of modifying genetic/epigenetic structure, intervening in the process of carcinogenesis $(8,9)$.

The length and extent of exposure to the many different EDs makes it difficult to evaluate the contribution of each agent. However, investigation on the modes of action may indicate the EDs that can act in an additive way on the same target following combined exposures. In this review, we evaluated the available evidence to clarify the controversial role of ED in female cancers.

Methods. The review was mainly based on a search of Medline using the key words: (('Endocrine Disruptors'[Mesh] OR endocrine disrupt*[ti] OR pesticide*[ti] OR 'Pesticides'[Mesh] OR ((Metals, Heavy[major] NOT ('Metals, Heavy/administration and dosage'[Mesh] OR 'Metals, Heavy/chemistry'[Mesh] OR 'Metals, Heavy/diagnostic use'[Mesh] OR 'Metals, Heavy/ pharmacokinetics'[Mesh] OR 'Metals, Heavy/pharmacology'[Mesh] OR 'Metals, Heavy/therapeutic use'[Mesh] OR radiotherapy[sh] OR radiation effects[sh])) OR METALS[TI] OR 'Metals, Heavy/adverse effects'[Mh] OR Metals, Heavy/ TOXICITY[MH]) OR 'bisphenol a'[ti] OR 'bisphenol A' [Supplementary Concept] OR dioxin*[ti] OR 'Dioxins'[Mesh] OR phtalate[ti]) AND (breast cancer[ti] OR ovarian cancer[ti] OR uterine cancer[ti] OR 'Breast Neoplasms'[MAJR] OR 'Genital Neoplasms, Female'[Mh]) AND (ita[la] OR eng[la] OR fre[la] OR ger[la] OR spa[la]) AND 2000:2014[dp]). Other data bases were used to retrieve literature, including Scopus and the Trip database.

\section{Difficulties involved in the study of the carcinogenic effect of endocrine disruptors}

The fraction of cancers attributable to ED are underestimated. Epidemiological findings do not identify a significant effect for each substance. However, a negative epidemiological finding may not be proof of absence of risk as shown in the reasons mentioned in Table I and below. 
ED carcinogenesis is complex, multifactorial and long lasting. ED cancer effects are methodologically difficult to study due to the multifactorial process of carcinogenesis and the time-lag between the inducing exposure and the clinical effect. Thus, ED doses at cancer diagnosis may not be associated with the biologically relevant exposure, which may have occurred much earlier. The first step of breast cancer initiation may occur as early as the fetal stage, while the cancer itself could clinically occur $\sim 50$ years later. The length of these different preclinical latency periods has been associated with exposure to other additional environmental carcinogens necessary for the complete development of the carcinogenic process $(10,11)$.

Lifelong exposure is difficult to evaluate. Two major relevant scenarios can be identified. Bioaccumulating ED, such dioxins, PCBs, perfluorinated chemicals or polybrominate flame retardants, constitute a lifelong body burden. Thus, biomonitoring through appropriate markers can provide a reliable proxy of the level of exposure experience through life, e.g., by dosing the parent substance or the metabolites (such as PCB-OH metabolites) in serum, plasma and cancer or adipose tissue biopsy (most persistent ED are lipophyllic). Non-persistent ED (most pesticides, phthalates, and bisphenol A) do not cause a body burden. Thus, measuring the level of the substance (or its metabolites) in body fluids may not reflect the possible relationship between exposure and slow-onset of diseases such as cancer. The best approach for non-persistent ED is to use case-control studies nested into biobanks. This approach might be difficult in developing countries. However, in these scenarios cancer burden and environmental pollution are on the increase (12).

Extremely low ED doses may interfere and gradually bioaccumulate. The risk from high-dose ED exposures, as in occupational studies, are well recognized (13). However, ED exposures in the general population are generally much lower than those employed in experimental studies, although exposure to almost every ED is plausible during the life span (including in uterus) through the diet and living environment. It is difficult to connect such exposure levels to cancer risk, due to insufficient information on the dose response relationships at such low doses, especially in the long term. However, accumulating evidence from human epidemiological and animal studies suggests that repeated and/or prolonged exposures to low ED doses are not devoid of adverse effects (14).

Endogenous steroid hormones have different phases of circulation. The active form of the hormone is free, the bio-available one is bound weakly to proteins such as albumin, whereas the inactive form is bound with high affinity to proteins such as sex hormone-binding globulin (SHBG). The three phases act as a buffering system, allowing the hormone to be accessible in the blood, but preventing the full dose of physiological hormones from acting. The potency of ED is several fold lower than that of physiological hormones, although serum levels in the general population, however low compared to toxicological studies, are also higher than hormones. Notably, with ED, there might be little or no fraction maintained in the inactive phase, because no physiological buffering system is in place. Thus, the entirety or majority of circulating EDs can be physiologically active, and even a low concentration of an ED can disrupt the natural balance of circulating endogenous hormones.

The overstimulation of hormone receptors, known as binding saturation, can downregulate the receptor, leading to reduced receptor density and a decrease in cells sensitivity to the hormone. This process often results in 'high-dose inhibition', an inverted U dose-response curve in which low doses have a different effect from high doses as they increase the response. Since low doses of endogenous hormones are present and fluctuating, small additions (or subtractions) to their actions may have a significant impact. For some EDs this suggests that even 'minor changes in consumer habits or industrial practices can have drastic effects on exposures' on the health significance of exposures (15).

At extremely high doses, chemicals can produce a number of toxic effects that obscure what would be most important for low-dose exposures. Therefore, occupational high-exposure studies, however important for their specific purpose, may not reflect the ED effects relevant to the general population. Accordingly, the Endocrine Society states that 'It cannot be assumed that high doses always provide information relevant to low-dose exposures, and it cannot be assumed that there is a threshold. The human population is chronically exposed to low doses of ED, which even further necessitates a 'no-threshold' approach to risk assessment of these chemicals' (1).

Another aspect that cannot be overlooked is the 'pulse' release of bioaccumulating ED. Lipophyllic ED are stored in the fat tissue over a long period of time causing a bioavailable burden with minimal daily doses as they bio-accumulate, even after the exposure ceases (16). Consequently, rapid mobilization of ED stored adipose tissue, for instance during drastic diets, may have an unwanted health impact (17).

ED have a combined exposure and a 'cocktail' effect. Exposure to several ED at the same time is the likely scenario. Even though pollutants have a multiform chemical nature as well as different molecular/biochemical mechanisms, they may nevertheless lead to the same adverse outcome pathway leading to additive or synergistic effects. The mixture of organochlorines, but not individual compounds, has been shown to enhance breast cancer cell proliferation (18). However, the mechanisms underlying cocktail effects, especially in real-life scenarios of long-term and low-level exposures, are far from being fully understood. Hormones exert widespread actions on multiple organs and systems, which are modulated by life stage and gender. In particular, hormone-relevant receptors or receptor isoforms may be differentially expressed in tissues or at different life stages. Moreover, EDs are imperfect ligands of hormone receptors and could interact with them in ways that do not perfectly replicate the actions of the endogenous hormones. Thus, it is possible that some EDs could cause a hormone receptor to act in a manner in which it would not normally act. The Endocrine Society states that 'This complexity causes difficulty when a static approach to toxicity through endocrine mechanisms driven by rigid guidelines is used to identify ED and manage risk' (1). Therefore, to consider the ED cocktail effects as a mere sum of hormone-like or hormone-antagonist may be an oversimplification that does not characterize the real human health hazards. The European Food Safety Authority (EFSA) 
has recently provided some advice on the cumulative toxicity of pesticides, since the frequent presence of multiple pesticide residues in foods is a major aspect of combined exposure to toxic chemicals. The EFSA states that, based on present knowledge, it is sound and conservative to assume that chemicals inducing the same effect in the same target organ, for example reduced thyroid function, act in an additive way, i.e., the chemicals sum up according to their respective potencies in inducing that effect and to their amounts. Notably, the EFSA points out that this additivity is expected to occur also when chemicals have similar effects but dissimilar mechanisms at molecular/biochemical level (19).

The ED can induce carcinogenic effects in vulnerable developmental periods. The studies, whether toxicological or epidemiological, on short-term exposures in adults are not suitable to set safe ED doses for the developing organism, because ED carcinogenic effects change over the lifetime of an individual. There are critical periods of development where the organism is more vulnerable to the ED cancer inducing and/or promoting effects, i.e., intrauterine tissue differentiation (late embryonic-early fetal stage) (20), puberty or the period prior to full-term pregnancy. In these relatively narrow windows, complex tissue growth and organization events occur: a hit during a susceptible window may have long-lasting consequences on the tissue. It is recognized that most ED do not exert direct genotoxic/carcinogenic actions. Instead, they may alter epigenetic regulation and/or cell-cell interactions, thus increasing the vulnerability of the organism to cancer (21).

The epigenetic effects of ED can also affect the future generations through environment-genome interactions. Developmental exposure to ED can alter gene expression by epigenetic changes. Thus, the impact can be potentially transferred to subsequent generations. The fungicide vinclozolin, an antagonist of the androgen receptor, may affect the male germ line epigenome in rodents promoting a pattern of transgenerational adult phenotypes that include disorders of reproductive (testis and prostate) and non-reproductive (kidney and immune system) target tissues, including cancer (22). Genomic alterations such as adduct formation, amplification of proto-oncogenes, chromosomal aberrations, DNA hypomethylation, and genetic instability are much more frequent in areas polluted by dioxins and some chemical elements with ED activities (e.g., cadmium and arsenic). Although an increased cancer risk may not be readily detectable at short term, it is biologically plausible that these genomic changes may affect cancer susceptibility in the future generations $(21,23)$.

Through their epigenetic effects, ED interfere with the long-term environment-genome interface, by means of a progressive and global hypo-methylation of DNA and a hyper-methylation of $\mathrm{CpG}$ islands (usually hypo-methylated) of the region promoter of tumor suppressor genes. Global hypomethylation of DNA would favor genomic and chromosomal instability. The selective hypermethylation $\mathrm{CpG}$ island, instead, acts by blocking the action of many tumor-suppressor genes that control cell proliferation, regulate apoptosis or the repair mechanisms (24).
Some individuals may have a particular susceptibility to $E D$ carcinogenesis. Individuals differ in susceptibility to the cancer effects of ED due to genetic polymorphism and epigenetic alterations. A given exposure is potentially carcinogenic only for a subgroup bearing a given polymorphism. Studies performed in the US have shown that PCB body burden is associated with increased risk of breast cancer in women with specific polymorphisms of the metabolizing enzyme CYP1A1. Notably, the polymorphisms involved were different between Caucasian and African-American women, indicating that these gene-environment interactions may have distinct features in different populations (25).

Hormone receptor concentration and affinity can vary among individuals: as they increases, significantly less ED is required to produce the same response. Another, less explored aspect of individual susceptibility is associated with nutrition: a lower or imbalanced intake of essential nutrients or antioxidants may lower the ability of the organism to cope with the ED effects (26). Thus, the field of nutrition (from nutrigenomics through to social and cultural habits) may deserve consideration in order to characterize highly susceptible population groups.

ED can also have indirect effects on carcinogen metabolism, immune system, oxidation and inflammation. The ED may have indirect effects by enhancing the transformation of environmental pro-carcinogens into carcinogens (27). It is well-recognized that several ED, particularly the polyhalogenated pollutants such as dioxins, PCBs, and DDT are inducers of the metabolizing enzymes of the P450 system. Other ED mechanisms may enhance the action of carcinogenic agents by impinging on oxidative stress and/or immune/inflammatory responses (27). ED exposure may cause the net production of reactive oxygen species (ROS) in tissues when antioxidant defense mechanisms are overwhelmed: the effects on many EDs (e.g., dioxins and PCB) are reduced by the concurrent intake of antioxidant vitamins such as vitamins $\mathrm{E}$ and $\mathrm{C}$ (26). The immune system is a recognized target of hormone regulation, thus, immune function could be modified by the ED that may interact with environmental inflammatory inducers involved in carcinogenesis (28-31).

The competing interests may limit research and public information on ED effects. An increased risk of cancer due to a specific ED, or ED group, may be underestimated or even remain undetected due to 'competing interests' or the so-called ‘business bias' (32,33).

\section{Models of ED carcinogenesis associated with female tumors}

According to experimental studies, the role of ED in endocrine-associated tumors of the female reproductive system are based on two models: i) the 'programming model', where the exposure to ED during the developmental life stages, from organogenesis through to puberty, alters the organization of the tissues making them more prone to carcinogenic insults later in life; and ii) the 'worsening model' where exposure to ED promotes the malignant evolution of precancerous or benign lesions. 
Since EDs are widespread in the environment, including in foods, the two models may occur during the life span of an individual. However, EDs have different modes of action $(5,34)$ and substances impinging on estrogen-regulated pathways are most relevant as regards a possible role on hormone-dependent tumors of women (35).

The programming model. Lipophilic ED stored prior to pregnancy in the adipose tissue can pass through the placentary barrier and expose the conceptus. During pregnancy there is also an inadvertent and continuous exposure of the mother-fetus dyad to ED released in the environment at very low and 'safe' (for adults) doses. When such 'low' doses filter the placental barrier, potentially irreversible epigenetic and somatic alterations may occur that could only become clinically detectable in adulthood, and that are well described in the literature (1,36-43).

Estrogen levels in the fetal environment have long-term consequences regarding the risk of developing breast cancer during adult life. Given the long latency period between exposure and effect, epidemiological studies designed to examine this hypothesis are required to use prenatal or neonatal markers of in utero estrogen exposure, because direct estrogen measurements are not available from birth records.

Dizygotic-twin pregnancy, which is associated with high estrogen levels, and pre-eclampsia, which is associated with low estrogen levels, are surrogates for high- and lowestrogen exposure, respectively. Dizygotic birth correlates with increased risk of breast cancer in the offspring while pre-eclampsia is associated with lowered risk.

During mammary gland development, breast epithelium may be particularly susceptible to environmental carcinogens $(44,45)$.

Toxicological studies conducted on rodents support the ED-induced altered programming of female reproductive functions, which has important consequences on the modulation of cancer risk. The prenatal exposure to substances acting through different mechanisms and on different pathways including the thyroid axis, may alter the long-term development of the female reproductive system (46-48) or the hypothalamic expression of neuropeptides relevant to female reproduction, such as oxytocin (49), in the absence of overt toxicity in the dams or pups. As regards predisposition to breast cancer, exposure of the organism to estrogens during its entire life is considered as a key risk factor, i.e., the more estrogen since early life, the higher the overall risk.

Breast cancer cell populations may arise from a sustained estrogen receptor (ER)-mediated proliferation of clusters of incompletely differentiated cells in the end buds. A different, albeit not necessarily alternative, hypothesis suggests that altered stromal-epithelial interactions lead to abnormal tissue remodeling (50). The end buds of the breast ducts, where cells are ER-rich, are the gland sites most responsive to estrogen stimulation during prenatal mammary organogenesis and peri-pubertal breast development. In this latter phase, ovarian estrogens stimulate cell division in the end buds that increasingly branch and elongate over time. Thus, the intrauterine life and peri-pubertal phase represent susceptible windows for factors that may alter tissue programming. Puberty is a life stage where profound endocrine and metabolic changes occur, affecting the reproductive as well as most other body systems. However, its role as a possible window specifically vulnerable to toxicants remains to be investigated (51).

In humans, premature puberty is associated with a higher risk of breast cancer and polycystic ovary syndrome, which is a risk factor for endometrial cancer. Besides rare genetic conditions, there is a global long-term trend towards earlier menarche, and especially towards earlier onset of breast development, due to a complexity of factors linked to diet, lifestyles and environment in which EDs may play a role (52). This trend towards earlier and more prolonged internal exposure to estrogens is a risk factor for breast cancer. Some EDs accelerate female puberty in rodents, such as PCB-altering steroidogenesis at the central nervous system level, ER $\alpha$ and ERB agonists, including the UV filter 4-methylbenzylidene camphor and the chlorinate insecticide lindane, respectively, and estrogen-active chemicals that do not bind to ERs such as the plasticizer butyl benzyl phthalate (34). By inducing earlier puberty onset such chemicals potentially indirectly increase the risk of breast and endometrial cancer.

The worsening model. Epidemiological findings indicate that exposure to some EDs may increase the severity of clinical conditions in women. For instance, higher concentrations of PCB in adipose tissue have been significantly associated with an increased risk of recurrence of breast cancer (53).

Most neoplasms of the breast in postmenopausal women require estrogens for their growth, as indicated by the relationship between ER-positive breast tumors and replacement therapy with estrogen-progesterone (54). The plasticizer bisphenol A induces the expression of the long non-coding RNA HOTAIR which leads to chromatin changes (histone methylation and acetylation) and activation of genes involved in breast cancer promotion (55).

A higher risk of estrogen-dependent endometrial cancer is associated with higher levels of endogenous estrogens, and this holds true for potentially precancerous endometrial hyperplasia. In a mouse strain vulnerable to endometrial neoplasia, loss of phosphatases and tensin homolog (PTEN) was the initiating lesion. However, transformation and progression processes were then accompanied by the altered expression levels of $>100$ ER- $\alpha$-regulated genes, mimicking a hyperestrogenic environment. Moreover, the promotion and progression of endometrial neoplasia are supported by an altered balance of steroid hormones, e.g., a high estradiol-low progesterone pattern as well as by other endocrine-related changes in cell and tissue metabolism, such as deregulation of insulin-like growth factor (IGF) pathways, activation of phosphorylating enzymes and increased prostaglandin E2. These changes occur in rodents as well as in humans, and indicate that epigenetic alterations in gene expression, e.g., of IGF, and/or a pro-inflammatory status may contribute to abnormal proliferation within the endometrial hyperplastic tissue. These changes have also been observed following ED exposure, although their possible relevance to an ED-related tumorpromoting effect, if any, has to be ascertained. For instance, the prenatal exposure to 'estrogenic' ED (methoxychlor and bisphenol A) alters the IGF expression in female rodents (56). The prostaglandin production in female reproductive tissues from animals is increased by DDT or the coplanar, dioxin-like 
Table II. How to prevent or limit pesticide exposure (Alavanja et al, modified) (7).

Dermal

1. Do not enter spaces where pesticides have been applied for the period specified on label instructions

2. Interrupt take-home pathways

3. Keep children and pets away from areas where pesticides were applied

4. Do not have pets enter the living areas of the home when soiled with pesticides until cleaned

5. Encourage family members exposed to pesticides to wash as soon as possible

6. Wash clothing soiled with pesticides separately from other laundry

Ingestion

1. Never store pesticides in cabinets with or near food

2. Always store pesticides in their original containers, complete with labels-instructions

3. Never transfer pesticides to soft drink bottles or other containers

4. Rinse fruits and vegetables with water. Scrub with a brush and peel them if possible

Inhalation/General

1. Do not stockpile pesticides. Purchase only what you need for immediate application

2. Follow the pesticide label directions for proper disposal

3. Report any symptoms possibly associated with pesticide exposure to your health care provider

4. If a close neighbor or someone else is applying pesticides outdoors near your home, stay indoors with your children and pets. Keep windows and doors closed

PCB 77 (57). Thus, whereas ED exposure is primarily relevant to the developing organism, its potential impact on vulnerable adults, such as women with benign or low-grade lesions, should receive attention.

\section{Prevention or limitation of ED exposure}

Since ED is not likely to be eliminated in the foreseeable future, cancer risk reduction depends on identifying the ED that are human carcinogens and limiting their exposure. Since the oncologic effects of the pollutants are poorly known, the principle of precaution should be used until each substance is proven to be safe. The most relevant behaviors to prevent ED exposure are the limitation of pesticide exposure and of use of plastic-type food contact materials (FCM) and the proper selection of drinking water, foods or daily activities.

Prevention or limitation of pesticide exposure. Evidence suggests that pesticides can be associated with excess cancer risk, including breast cancer, for those applying the pesticide and those who are merely bystanders to the application. Until a more complete understanding of pesticide carcinogenesis is achieved, health care providers should emphasize the importance of minimizing personal exposure (7). A practical guideline for preventing or limiting pesticide exposure is provided in Table II.

Limiting plastic-type food contact materials. Food contact materials are an underestimated source of human exposure to EDs across the entire human life span.

Plastic-type food contact materials leach mixtures of substances at low concentrations. These mixtures comprise monomers, additives, manufacturing aids, side-products, impurities, printing inks, adhesives, and other compounds. Not all of the components of the whole migrate mixture have been identified. The toxicity of this migrate in foods is of concern because it is this mixture that consumers are exposed to rather than the single food contact materials substances. Women of childbearing age and during pregnancy are a very sensitive population group requiring much more attention. In overweight and obese persons a change in the metabolism of $\mathrm{ED}$ is observed and lipophilic ED storage is greater. Heating increases leakage and contamination (58).

Thus, encouraging the consumption of fresh foods, avoiding canned food and plastic packaging for storing and reheating foods and beverages is vital.

Limitation of ED contaminants in drinking water, foods or daily life. A practical guideline for preventing or limiting ED exposure can be drawn from the Italian website on ED (http:// www.iss.it/inte) or from the Italian 'Previeni' project (www. iss.it/prvn) (Table III).

It is advisable to use uncontaminated or less ED contaminated water. Private wells appear to be less safe.

Feminization of aquatic animals has raised concerns regarding estrogenic compounds in water supplies and the potential for these chemicals to reach drinking water.

\section{Considerations for future investigations}

Data available on breast tumorigenesis are more abundant than for endometrial cancer, while ovarian cancer has been little explored and needs more attention. Additionally, only a limited number of potentially relevant ED have been investigated, albeit with different modes of action. The available data outline the importance of the programming model, and therefore of exposures during windows of heightened vulnerability during in utero and peripubertal development. The ability of ED to exacerbate the clinical outcome of hormone-dependent cancer is supported by mechanistic data, but is more difficult to assess 
Table IIIa. Decalogue (ISS, modified) (p. 198).

A, General

\section{Limit or avoid}

1. Do not reuse worn or disposable plastic containers for food and beverages

2. Restrict the use of non-stick cooking utensils if the coating is worn

3. Use wax paper or film in contact with food only in accordance with the manufacturer's instructions. Always read the labels

4. While cooking foods properly ventilate premises and use extractor hoods

5. Limit the combustion of incense and candle smoke and avoid smoking in the environment where you live

6. Replace torn wrappers and/or worn objects with foam padding such as car seats and mattresses

7. Restrict the use of clothing with water or stain repellent treatments

8. Avoid the consumption of foods in carbonized materials. Remove burnt parts from foods or burned parts and restrict the use of smoked foods

9. When choosing materials for the house restrict to the use of soft PVC containing DEHP

10. Prevent dust accumulation in enclosed spaces
Favor or replace

Use plastic containers intact and only for the uses specified by the manufacturer

Use cookware intact and suitable for food contact

Ensure frequent replacement of indoor air

Favor clothing readily identifiable sources and composition

B, Decalogue for children (ISS, modified) (198).

Limit or avoid

1. Prevent dust accumulation and air stagnation in the environments where little children crawl or play on the ground

2. If you have DEHP-containing PVC flooring on which children play, use an untreated-fiber carpet

3. Restrict the use of child clothing with water or stain repellent treatments

4. Do not use mattresses covered with waterproof canvas not complying with the current standards and prevent mattress coverings made with soft DEHP-containing PVC

5. Use untreated fiber linings if you have changing tables and/or strollers covered in soft DEHP-containing PVC; as a general rule prevent children from ingesting $\mathrm{PVC}$

6. Use intact containers to warm up milk, beverages and baby foods and only according to the manufacturer's instructions

7. Allow hot liquids to cool off before transfering them into plastic containers not intended for use at high temperatures

8. Wash baby bottles and other containers thoroughly after sterilization; do not use polycarbonate baby bottles (no longer allowed)

9. Accustom the children to consume fresh seasonal foods; rinse canned fruits and vegetables before consumption

10. Avoid the consumption of foods with carbonized or burned parts

\section{Favor or replace}

Clean the environment adequately and regularly and properly maintain the vacuum cleaners (cleaning filters and collection chambers, replacing bags, if any)
Warrant the replacement of air in enclosed rooms and clean adequately and regularly the environment and properly maintain the vacuum cleaners (cleaning filters and collection chambers, replacing bags, if any)

Favor clothing from readily identifiable sources and composition

When cooking food for children, favor methods that preserve the content of water-soluble vitamins

(e.g., steaming) 
using present in vivo models. Chemicals that may raise concern due to their carcinogenic potential (mainly because showing genotoxic activity) are tested by chronic carcinogenicity bioassays in rodents. However, whether these studies are actually useful to evaluate the potential for promoting hormone-related cancers is indeterminable. The standard carcinogenity bioassay protocols do not cover the in utero development and might even start after puberty. Whereas over 200 chemicals were identified as mammary gland carcinogens in carcinogenicity bioassays, these results may reflect more the generic ability of a substance to induce tumors in tissues where it reaches sufficient active concentrations. Understanding the modes of action at the level of target issues and endocrine regulation may be more relevant to hormone-dependent cancers than performing standard cancer bioassays using special strains or dosing schedules. Investigating modes of action may provide other useful information as well, such as the evaluation of whether an observed effect may be relevant to humans from the toxicodynamic or toxicokinetic standpoints. Besides the classical endocrine-related mechanisms (e.g., interactions with nuclear receptors), investigation concerning other potential ED targets in relation to cancer such as signaling molecules (e.g., kisspeptins) and disturbances of specific methylation processes is required. The epigenetic effects can be underestimated in the present literature and should be the main area of investigation and assessing of ED due to the potential long-term and transgenerational effects.

\section{Conclusion}

Research on the effects of ED on female neoplasms is difficult due to methodological limitations as reviewed in this study. Over 85,000 chemicals are among the common daily exposures to individuals, but we still have limited data on which are ED that could affect risk of disease. The number of potential EDs introduced in the environment is increasing. However, the carcinogenetic effect of only a few of these is known and mostly by experimental or in vitro data. Cancer appears to be a more complex process than previously thought. An increasing role of alterations in stroma or extracellular matrix induced by persistent signals arising from the microenvironment has been identified, inducing epigenetic and genetic modifications in tissue stem cells that can lead to cancer (59).

Thus new oncologic challenges (60) are currently being addressed and future cancer treatment should take into account ED. As fundamental changes in lifestyle can effectively counter the bio-accumulation of some EDs (61) identifying treatment is imperative. However, adequate clinical research is time consuming and requires numerous resources. ED can also affect the health of future generations, through epigenetic mechanisms. Thus, current evidence of the carcinogenic effect of ED for female cancers is sufficient to require precautionary actions to limit exposure.

\section{References}

1. Zoeller RT, Brown TR, Doan LL, Gore AC, Skakkebaek NE, Soto AM, Woodruff TJ and Vom Saal FS: Endocrine-disrupting chemicals and public health protection: a statement of principles from The Endocrine Society. Endocrinology 153: 4097-4110, 2012.
2. Kavlock RJ, Daston GP, DeRosa C, Fenner-Crisp P, Gray LE, Kaattari S, Lucier G,Luster M, Mac MJ, Maczka C, et al: Research needs for the risk assessment of health and environmental effects of endocrine disruptors: a report of the US EPA-sponsored workshop. Environ Health Perspect 104 (Suppl 4): 715-740, 1996.

3. Damstra T, Barlow S, Bergman A, Kavlock RJ and van derKraak G (eds): Global Assessment of the State-of-the-Science of Endocrine Disruptors. International Programme on Chemical Safety. World Health Organization, Geneva, 2002.

4. European Commission: European workshop on the impact of endocrine disruptors on human health and wildlife. In: Environment and Climate Research Programme DX. European Commission, Brussels, 1996.

5. Mantovani A: Risk assessment of endocrine disrupters: the role of toxicological studies. Ann NY Acad Sci 1076: 239-252, 2006.

6. Boffetta P, Couto E, Wichmann J, Ferrari P, Trichopoulos D, Bueno-de-Mesquita HB, van Duijnhoven FJ, Büchner FL, Key T, Boeing $\mathrm{H}$, et al: Fruit and vegetable intake and overall cancer risk in the European Prospective Investigation into Cancer and Nutrition (EPIC). J Natl Cancer Inst 102: 529-537, 2010.

7. Alavanja MC, Ross MK and Bonner MR: Increased cancer burden among pesticide applicators and others due to pesticide exposure. CA Cancer J Clin 63: 120-142, 2013.

8. Clapp RW, Jacobs MM and Loechler EL: Environmental and occupational causes of cancer: new evidence 2005-2007. Rev Environ Health 23: 1-37, 2008.

9. Tomatis L: Role of experimental and epidemiological evidence of carcinogenicity in the primary prevention of cancer. Ann Ist Super Sanita 42: 113-117, 2006.

10. Loeb KR and Loeb LA: Significance of multiple mutations in cancer. Carcinogenesis 21: 379-385, 2000.

11. Michor F, Iwasa Y and Nowak MA: Dynamics of cancer progression. Nat Rev Cancer 4: 197-205, 2004.

12. Vineis $P$ and Xun W: The emerging epidemic of environmental cancers in developing countries. Ann Oncol 20: 205-212, 2009.

13. Weichenthal S, Moase C and Chan P: A review of pesticide exposure and cancer incidence in the Agricultural Health Study cohort. Environ Health Perspect 118: 1117-1125, 2010.

14. Irigaray $\mathrm{P}$ and Belpomme D: Basic properties and molecular mechanisms of exogenous chemical carcinogens. Carcinogenesis 31: 135-148, 2010.

15. Vandenberg LN, Colborn T, Hayes TB, Heindel JJ, Jacobs DR Jr, Lee DH, Shioda T, Soto AM, vom Saal FS, Welshons WV, et al: Hormones and endocrine-disrupting chemicals: low-dose effects and nonmonotonic dose responses. Endocr Rev 33: 378-455, 2012.

16. Diamanti-Kandarakis E, Bourguignon JP, Giudice LC, Hauser R, Prins GS, Soto AM, Zoeller RT and Gore AC: Endocrine-disrupting chemicals: an Endocrine Society scientific statement. Endocr Rev 30: 293-342, 2009.

17. Hue O, Marcotte J, Berrigan F, Simoneau M, Doré J, Marceau P, Marceau S, Tremblay A and Teasdale N: Increased plasma levels of toxic pollutants accompanying weight loss induced by hypocaloric diet or by bariatric surgery. Obes Surg 16: 1145-1154, 2006.

18. Payne J, Scholze M and Kortenkamp A: Mixtures of four organochlorines enhance human breast cancer cell proliferation. Environ Health Perspect 109: 391-397, 2001.

19. No authors listed: Scientific opinion on the relevance of dissimilar mode of action and its appropriate application for cumulative risk assessment of pesticides residues in food. EFSA 11: 3472, 2013.

20. Tomatis L: Prenatal exposure to chemical carcinogens and its effect on subsequent generations. Natl Cancer Inst Monogr 51: 159-184, 1979.

21. Shapiro JA: Revisiting the central dogma in the 21st century. Ann NY Acad Sci 1178: 6-28, 2009.

22. Skinner MK and Anway MD: Epigenetic transgenerational actions of vinclozolin on the development of disease and cancer. Crit Rev Oncog 13: 75-82, 2007.

23. Salnikow K and Zhitkovich A: Genetic and epigenetic mechanisms in metal carcinogenesis and cocarcinogenesis: nickel, arsenic, and chromium. Chem Res Toxicol 21: 28-44, 2008.

24. Eden A, Gaudet F, Waghmare A and Jaenisch R: Chromosomal instability and tumors promoted by DNA hypomethylation. Science 300: 455, 2003.

25. Li Y, Millikan RC, Bell DA, Cui L, Tse CK, Newman B and Conway K: Polychlorinated biphenyls, cytochrome P450 1A1 (CYP1A1) polymorphisms, and breast cancer risk among African American women and white women in North Carolina: a population-based case-control study. Breast Cancer Res 7: R12-R18, 2005. 
26. Baldi F and Mantovani A: A new database for food safety: EDID (Endocrine disrupting chemicals - Diet Interaction Database). Ann Ist Super Sanita 44: 57-63, 2008.

27. Loeb LA and Harris CC: Advances in chemical carcinogenesis: a historical review and prospective. Cancer Res 68: 6863-6872, 2008.

28. Valko M, Rhodes CJ, Moncol J, Izakovic M and Mazur M: Free radicals, metals and antioxidants in oxidative stress-induced cancer. Chem Biol Interact 160: 1-40, 2006.

29. Pikarsky E, Porat RM, Stein I, Abramovitch R, Amit S, Kasem S, Gutkovich-Pyest E, Urieli-Shoval S, Galun E and Ben-Neriah Y: NF-kappaB functions as a tumour promoter in inflammation-associated cancer. Nature 431: 461-466, 2004.

30. de Visser KE and Coussens LM: The inflammatory tumor microenvironment and its impact on cancer development. Contrib Microbiol 13: 118-137, 2006.

31. van Kempen LC, de Visser KE and Coussens LM: Inflammation, proteases and cancer. Eur J Cancer 42: 728-734, 2006.

32. Gennaro V and Tomatis L: Business bias: how epidemiologic studies may underestimate or fail to detect increased risks of cancer and other diseases. Int J Occup Environ Health 11: $356-359,2005$

33. Kjaergard LL and Als-Nielsen B: Association between competing interests and authors' conclusions: epidemiological study of randomised clinical trials published in the BMJ. BMJ 325: 249 , 2002.

34. Maranghi F and Mantovani A: Targeted toxicological testing to investigate the role of endocrine disrupters in puberty disorders. Reprod Toxicol 33: 290-296, 2012.

35. Caserta D, Maranghi L, Mantovani A, Marci R, Maranghi F and Moscarini M: Impact of endocrine disruptor chemicals in gynaecology. Hum Reprod Update 14: 59-72, 2008.

36. Barr DB, Bishop A and Needham LL: Concentrations of xenobiotic chemicals in the maternal-fetal unit. Reprod Toxicol 23: 260-266, 2007

37. Cohn BA, Wolff MS, Cirillo PM and Sholtz RI: DDT and breast cancer in young women: new data on the significance of age at exposure. Environ Health Perspect 115: 1406-1414, 2007.

38. Hilakivi-Clarke L and de Assis S: Fetal origins of breast cancer Trends Endocrinol Metab 17: 340-348, 2006.

39. Cook JD, Davis BJ, Cai SL, Barrett JC, Conti CJ and Walker CL: Interaction between genetic susceptibility and early-life environmental exposure determines tumor-suppressor-gene penetrance. Proc Natl Acad Sci USA 102: 8644-8649, 2005.

40. Hilakivi-Clarke L: Nutritional modulation of terminal end buds: its relevance to breast cancer prevention. Curr Cancer Drug Targets 7: 465-474, 2007.

41. Warri A, Saarinen NM, Makela S and Hilakivi-Clarke L: The role of early life genistein exposures in modifying breast cancer risk. Br J Cancer 98: 1485-1493, 2008

42. Butler Walker J, Seddon L, McMullen E, Houseman J, Tofflemire K, Corriveau A, Weber JP, Mills C, Smith S and Van Oostdam J: Organochlorine levels in maternal and umbilical cord blood plasma in Arctic Canada. Sci Total Environ 302: 27-52, 2003.

43. Soto AM, Vandenberg LN, Maffini MV and Sonnenschein C: Does breast cancer start in the womb? Basic Clin Pharmacol Toxicol 102: 125-133, 2008.

44. Fenton SE: Endocrine-disrupting compounds and mammary gland development: early exposure and later life consequences. Endocrinology 147 (Suppl): S18-S24, 2006.

45. Rudel RA, Fenton SE, Ackerman JM, Euling SY and Makris SL: Environmental exposures and mammary gland development: state of the science, public health implications, and research recommendations. Environ Health Perspect 119: 1053-1061, 2011.
46. Maranghi F, Rescia M, Macrì C, Di Consiglio E, De Angelis G, Testai E, Farini D, De Felici M, Lorenzetti S and Mantovani A: Lindane may modulate the female reproductive development through the interaction with ER-beta: an in vivo-in vitro approach. Chem Biol Interact 169: 1-14, 2007.

47. Maranghi F, Tassinari R, Moracci G, Macrì C and Mantovani A: Effects of a low oral dose of diethylstilbestrol (DES) on reproductive tract development in F1 female CD-1 mice. Reprod Toxicol 26: 146-150, 2008.

48. Maranghi F, De Angelis S, Tassinari R, Chiarotti F, Lorenzetti S, Moracci G, Marcoccia D, Gilardi E, Di Virgilio A, Eusepi A, et al: Reproductive toxicity and thyroid effects in Sprague Dawley rats exposed to low doses of ethylenethiourea. Food Chem Toxicol 59: 261-271, 2013.

49. Tait S, Ricceri L, Venerosi A, Maranghi F, Mantovani A and Calamandrei G: Long-term effects on hypothalamic neuropeptides after developmental exposure to chlorpyrifos in mice. Environ Health Perspect 117: 112-116, 2009.

50. Soto AM and Sonnenschein C: Environmental causes of cancer: endocrine disruptors as carcinogens. Nat Rev Endocrinol 6: 363-370, 2010.

51. Mantovani A and Fucic A: Puberty dysregulation and increased risk of disease in adult life: possible modes of action. Reprod Toxicol 44: 15-22, 2014

52. Mantovani A: Endocrine disruptors and puberty disorders from mice to men (and women). In: Endocrine Disruptors and Puberty. Kandarakis ED and Gore AC (eds). Contemporary Endocrinology Part 1, Springer, pp119-137, 2012.

53. Muscat JE, Britton JA, Djordjevic MV, Citron ML, Kemeny M, Busch-Devereaux E, Pittman B and Stellman SD: Adipose concentrations of organochlorine compounds and breast cancer recurrence in Long Island, New York. Cancer Epidemiol Biomarkers Prev 12: 1474-1478, 2003.

54. Key T, Appleby P, Barnes I and Reeves G; Endogenous Hormones and Breast Cancer Collaborative Group: Endogenous sex hormones and breast cancer in postmenopausal women: reanalysis of nine prospective studies. J Natl Cancer Inst 94: 606-616, 2002

55. Bhan A, Hussain I, Ansari KI, Bobzean SA, Perrotti LI and Mandal SS: Bisphenol-A and diethylstilbestrol exposure induces the expression of breast cancer associated long noncoding RNA HOTAIR in vitro and in vivo. J Steroid Biochem Mol Biol 141: 160-170, 2014.

56. Zama AM and Uzumcu M: Targeted genome-wide methylation and gene expression analyses reveal signaling pathways involved in ovarian dysfunction after developmental EDC exposure in rats. Biol Reprod 88: 52, 2013.

57. Wrobel MH,Mlynarczuk J and Kotwica J: The effect of DDT and its metabolite (DDE) on prostaglandin secretion from epithelial cells and on contractions of the smooth muscle of the bovine oviduct in vitro. Toxicol Appl Pharmacol 259: 152-159, 2012.

58. Muncke J: Endocrine disrupting chemicals and other substances of concern in food contact materials: an updated review of exposure, effect and risk assessment. J Steroid Biochem Mol Biol 127: 118-127, 2011

59. Burgio E and Migliore L: Towards a systemic paradigm in carcinogenesis: linking epigenetics and genetics. Mol Biol Rep 42: 777-790, 2015

60. Berretta M, Di Francia R and Tirelli U: The new oncologic challenges in the 3rd millennium. Editorial WCRJ 1: e133, 2014.

61. Del Buono A, D'Orta A, Del Buono R, Del Buono MG, De Monaco A and Marullo MN: Relationship between diet and heavy metals in high risk of the environmental toxicity areas. Implication for cancer prevention. WCRJ 1: e411, 2014. 